\begin{tabular}{|c|l|}
\hline Title & A nalysis and control of excess leakage currents in nitride based Schottky diodes based on thin surface barrier model \\
\hline Author(s) & Kotani, Junji; Hashizume, Tamotsu; Hasegawa, Hideki \\
\hline Citation & $\begin{array}{l}\text { Journal of Vacuum Science \& Technology B: Microelectronics and Nanometer Structures, 22(4), 2179-2189 } \\
\text { https:/doi.org/10.1116/1.1771678 }\end{array}$ \\
\hline Issue Date & 200408 20 \\
\hline Doc URL & http:/hdl.handle.net/2115/5803 \\
\hline Type & article \\
\hline File Information & JV STB22-4.pdf \\
\hline
\end{tabular}

Instructions for use 


\title{
Analysis and control of excess leakage currents in nitride-based Schottky diodes based on thin surface barrier model
}

\author{
Junji Kotani, ${ }^{\text {a) }}$ Tamotsu Hashizume, and Hideki Hasegawa \\ Research Center for Integrated Quantum Electronics (RCIQE) and Graduate School of Electronics and \\ Information Engineering, Hokkaido University, Sapporo 060-8628, Japan
}

(Received 18 January 2004; accepted 3 April 2004; published 20 August 2004)

\begin{abstract}
Using a rigorous computer simulation program for current transport through a Schottky barrier with an arbitrary potential profile, the leakage current mechanism in GaN and AlGaN Schottky diodes was investigated on the basis of the thin surface barrier (TSB) model recently proposed by the authors' group. Computer simulation assuming various possible defect density distributions was carried out to reproduce the measured temperature dependent current voltage $(I-V)$-temperature characteristics of the GaN and AlGaN Schottky diodes which showed excessive reverse leakage. By assuming exponentially decaying distributions from surface for defect donors with energy depth of $0.25 \mathrm{eV}$ for $\mathrm{GaN}$ and $0.37 \mathrm{eV}$ for $\mathrm{Al}_{0.15} \mathrm{Ga}_{0.85} \mathrm{~N}, I-V$ curves measured by our group as well as reported in the literatures were almost completely reproduced both in forward and reverse direction over a wide temperature range. The defect donors are proposed to be $\mathrm{N}$ vacancies or their related complexes that are formed during metal deposition. The result confirms the validity of the TSB model. From the viewpoint of the TSB model, attempts were also made to suppress leakage currents. It was found that a low-energy electrochemical metal deposition process and a metalinsulator-semiconductor Schottky structure using an ultrathin $\mathrm{Al}_{2} \mathrm{O}_{3}$ film by electron cyclotron resonance oxidation of $\mathrm{Al}$ film were remarkably effective in reducing excess leakage currents due to reduction of defect deep donors. (C) 2004 American Vacuum Society. [DOI: 10.1116/1.1771678]
\end{abstract}

\section{INTRODUCTION}

Formation of high-quality Schottky barrier contacts on III-V nitrides is essentially important for their application to devices such as high frequency/high power heterostructure field effect transistors (HFETs), metal-semiconductor FETs, power rectifiers, ultraviolet photodetectors, and various gas sensors. In particular, smallness of reverse-bias leakage currents of the Schottky barrier is a major concern in such devices from the viewpoints of precise depletion edge control, operation stability and reliability, power consumption, and noise performance. However, Schottky diodes formed on $\mathrm{GaN}$ and $\mathrm{AlGaN}$ usually suffer from excess reverse leakage currents that are many orders of magnitude larger than the prediction of the thermionic emission (TE) model. ${ }^{1-8}$ This causes serious problems such as the off-state leakage in FETs $^{9}$ and the dark current in various kinds of detectors. We have also proposed recently that surface defects causing large leakage in Schottky gates are also responsible for the so-called current collapse in AlGaN/GaN HFETs. ${ }^{8}$

Although numerous studies have been made on $\mathrm{GaN}$ based Schottky diodes having various kinds of barrier metals, they focused, in most cases, on the forward current transport and only limited attempts have so far been made to explain the mechanism for large reverse leakage currents. For example, Yu et al. ${ }^{10}$ and Miller et al. ${ }^{11}$ calculated tunneling currents through $\mathrm{GaN}$ and $\mathrm{AlGaN}$ Schottky barriers, assuming a triangular Schottky barrier. However, in their calculation, they had to use unreasonably large donor densities

\footnotetext{
a) Author to whom correspondence should be addressed; electronic mail: kotani@rciqe.hokudai.ac.jp
}

that are more than 1 order of magnitude larger than the actual doping concentration in order to reproduce the observed temperature $(T)$-dependent current-voltage $(I-V)$ curves. Thus, they had to suggest some other processes such as defectassisted tunneling which enhances leakage currents. Although several other groups also suggested contribution of the trap-assisted tunneling transport to excess reverse leakage currents, ${ }^{3,12}$ such transport requires either a multistep tunneling process with unrealistically large electron capture cross sections of defects, or presence of a energy continuum formed by defects with a significantly wide energy band width throughout the depletion region of the semiconductor. Thus, presence of such a transport process is far from having been well established.

On the other hand, we have recently proposed an entirely different model called the thin surface barrier (TSB) model $^{7}$ for both forward and reverse current transport in $\mathrm{GaN}$ Schottky barriers. Here, it is assumed that the width of the Schottky barrier is reduced due to the presence of unintentional surface defect donors, and that thermionic field emission (TFE) or field emission (FE) through the resultant thin surface barrier gives the major current leakage path in both forward and reverse directions. This model could explain the temperature dependences of the measured $I-V$ curves systematically. However, detailed quantitative agreements between theory and experiment were not entirely satisfactory, and this was thought to be due to use of approximate analytical formulas that were obtainable only for the case of a simple rectangular distribution of shallow defect donors.

The purpose of this article is to further confirm the validity of the TSB model for GaN and AlGaN Schottky barriers 


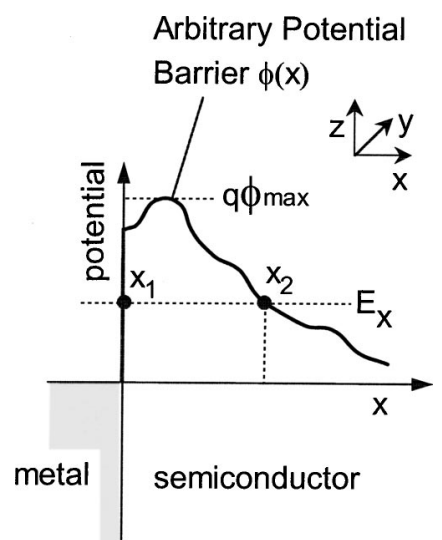

FIG. 1. Schottky barrier with an arbitrary potential profile considered in the present simulation.

by a rigorous computer simulation. Limited attempts to reduce excess leakage currents were also made on the basis of an understanding. For this purpose, a simulation program for an arbitrary distribution of defect donors was developed. It is shown that deep defect donors with particular energy depths and exponentially decaying density distributions from the surface can reproduce the observed $I-V$ characteristics of our GaN and AlGaN Schottky diodes as well as others almost completely over a wide temperature range. The defect donors are proposed to be $\mathrm{N}$ vacancies or their related complexes that are formed during metal deposition. Additionally, a low-energy electrochemical metal deposition process and a surface passivation process using an ultrathin $\mathrm{Al}_{2} \mathrm{O}_{3}$ layer were found to be very effective in suppressing formation of surface defect donors and reducing reverse leakage currents.

\section{NUMERICAL SIMULATION METHOD OF $I-V-T$ CHARACTERISTICS}

\section{A. Current transport in a Schottky barrier with an arbitrary potential profile}

Let us start by considering current transport in a Schottky barrier with an arbitrary potential barrier shown in Fig. 1 in a general way. The infinitesimal number of electrons having a total energy, $E$ in the infinitesimal volume $d k_{x} d k_{y} d k_{z}$ in the $k$ space may move from semiconductor to metal or from metal to semiconductor through the Schottky barrier. These flows can be expressed by the following equations, respectively:

$$
\begin{aligned}
& d n_{s \rightarrow m}=2 f_{s}(E)\left[1-f_{m}(E)\right] T\left(E_{x}\right)\left(\frac{1}{2 \pi}\right)^{3} d k_{x} d k_{y} d k_{z}, \\
& d n_{m \rightarrow s}=2 f_{m}(E)\left[1-f_{s}(E)\right] T\left(E_{x}\right)\left(\frac{1}{2 \pi}\right)^{3} d k_{x} d k_{y} d k_{z} .
\end{aligned}
$$

Here, it is assumed that energy and momentum are conserved during transport and that the transport probability for an electron is given by $T\left(E_{x}\right)$ as a function of the $x$ component of energy, $E_{x}=\hbar^{2} k_{x}^{2} / 2 m^{*}$ where $k_{x}$ is the wave number normal to the barrier, $\hbar$ is the reduced Planck's constant, and $m^{*}$ is the effective mass. $k_{y}$ and $k_{z}$ are the wave number parallel to the Schottky barrier, and $f_{s}(E)$ and $f_{m}(E)$ are the FermiDirac distribution functions for semiconductor and metal, respectively. Since only electrons that have a negative or positive group velocity component can go into the Schottky barrier from the semiconductor and from the metal side, respectively, and contribute to the current, the current from semiconductor to metal through the Schottky barrier region can be expressed by the following general expression:

$$
\begin{aligned}
J= & \int_{-\infty}^{\infty} \int_{-\infty}^{\infty} \int_{0}^{\infty} \frac{2 q}{\hbar}\left(\frac{1}{2 \pi}\right)^{3}\left[f_{s}(E)-f_{m}(E)\right] T\left(E_{x}\right) \\
& \times\left(\frac{d E_{x}}{d k_{x}}\right) d k_{x} d k_{y} d k_{z} .
\end{aligned}
$$

By converting the double integral with respect to $k_{y}$ and $k_{z}$ to an integral with respect to the parallel component energy, $E_{p}=\left(\hbar^{2} / 2 m^{*}\right)\left(k_{y}^{2}+k_{z}^{2}\right)$, Eq. (2) reduces to

$$
\begin{aligned}
J= & \frac{4 \pi q m^{*}}{h^{3}} \int_{0}^{\infty} T\left(E_{x}\right) \int_{0}^{\infty}\left[f_{s}\left(E_{p}+E_{x}\right)\right. \\
& \left.-f_{m}\left(E_{p}+E_{x}\right)\right] d E_{p} d E_{x} .
\end{aligned}
$$

This is a very general basic expression for current transport in a Schottky barrier first derived for field emission into vacuum by Stratton. ${ }^{13}$ Presently it is often used in the device simulators. If we only consider electrons which have a sufficiently large $x$ component energy to go over the Schottky barrier, and we assume that $T\left(E_{x}\right)=1$ for them, ignoring possible quantum reflection, and that $T\left(E_{x}\right)=0$ for the rest of electrons, Eq. (3) reduces to the standard equation for TE transport.

In the case where tunneling transport through the barrier becomes very important as in the case of the present study, the simplest way to deal with it is to use the conventional (WKB) approximation, although a more elaborate and rigorous approach using the transition matrix may also be used. According to the Wentzel-Kramers-Brillouin approximation, the tunneling probability $T\left(E_{x}\right)$ for an electron having an $x$ component of energy, $E_{x}$, is given by

$$
T\left(E_{x}\right)=\exp \left[-2 \frac{\sqrt{2 m^{*}}}{\hbar} \int_{x_{1}}^{x_{2}} \sqrt{\phi(x)-E_{x}} d x\right],
$$

where $\phi(x)$ is the potential function, and $x_{1}$ and $x_{2}$ are classical turning points shown in Fig. 1.

Historically, Padovani and Stratton ${ }^{14}$ studied the tunneling current transport through a Schottky barrier formed on a highly doped material by combining Eqs. (3) and (4), and derived approximate analytical formulas for $I-V$ characteristics. The background physics was the following. Since $T\left(E_{x}\right)$ is an exponentially increasing function of $E_{x}$ as seen in Eq. (4), and $f_{m}\left(E_{x}\right)$ is an exponentially decreasing function, the integrand in Eq. (3) forms a Gaussian peak for tunneling at a certain energy whose energy position is temperature dependent. At a sufficiently low temperature, this peak takes place at the Fermi level, leading to the so-called FE at 
the Femi level. On the other hand, at elevated temperatures, the peak occurs above the Fermi level, leading to the TFE. From such a consideration, Padovani and Stratton were able to derive approximate analytical formulas based on the Taylor expansion of the integrand of the above Eq. (3) around the energy position for the Gaussian peak.

However, such an analytical treatment is only possible for uniformly doped semiconductors. Therfore, in the present article, $I-V$ characteristics were numerically calculated using Eqs. (3) and (4) for various possible distributions of nearsurface defect donors on the basis of the TSB model. For this purpose, a computer program to calculate Eqs. (3) and (4) was developed. The potential profile of the Schottky barrier was calculated by solving the Poisson equation selfconsistently, using a simulation program previously developed by our group. Unlike the analytical treatment by Padovani and Stratton, ${ }^{14}$ our numerical calculation includes contribution of thermionic emission by putting $T\left(E_{x}\right)=1$ for electrons which can go over the Schottky barrier. Such a TE transport becomes dominant at high temperatures. Thus, our approach takes into account all the possible current transport mechanisms automatically.

\section{B. Comparison with previous analytical formulas and with experiment on GaN}

First, the validity of the previous analytical formulas by Padovani and Stratton ${ }^{14}$ was investigated for uniformly doped semiconductors. For this, we calculated $I-V$ characteristics of a Schottky diode for various doping densities and temperatures by our computer program, and compared the results with those by the analytical formulas. For the forward bias region, both methods gave nearly the same $I-V$ characteristics. In particular, analytical formulas included the following well known formula for the ideality factor $n_{F}$ for the forward TFE/TE transport

$$
n_{F}=\left(E_{00} / k T\right) \operatorname{coth}\left(E_{00} / k T\right),
$$

with

$$
E_{00}=2 q\left[N_{D} / 2 \epsilon\right]^{1 / 2} \hbar / 2\left(2 m^{*}\right)^{1 / 2},
$$

where $N_{D}$ is the bulk doping density of the semiconductor. In Fig. 2(a), calculated values of $n_{F}$ using Eq. (5) are shown by solid curves and those by our computer program are shown by symbols. The Schottky barrier height $(\mathrm{SBH})$ was assumed to be $0.85 \mathrm{eV}$. A very good agreement is seen here.

For the reverse leakage currents, calculated curves by the analytical formulas of Padovani and Stratton and those by the computer program are shown in Fig. 2(b) by solid and dashed curves, respectively, for a Schottky diode with $\mathrm{SBH}$ $=0.85 \mathrm{eV}$ and a doping density of $N_{D}=1 \times 10^{17} \mathrm{~cm}^{-3}$. Again, good agreements are seen. Agreements became somewhat poorer as the doping density was increased into the $10^{18} \mathrm{~cm}^{-3}$ range due to the following unfavorable features of the analytical formulas for the reverse currents. Namely, two different analytical formulas were derived: one for the large reverse bias range where transport is strongly by the FE mechanism and one for the small reverse bias range where

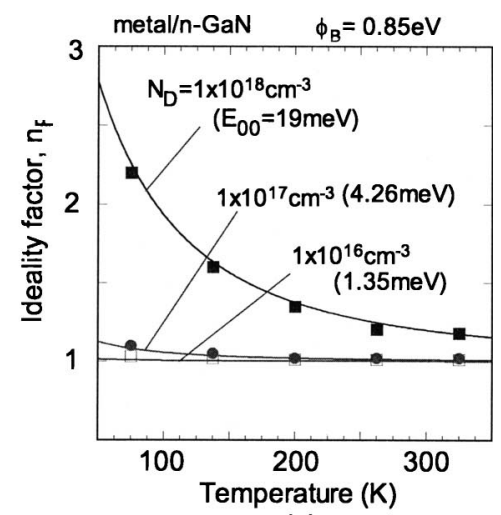

(a)

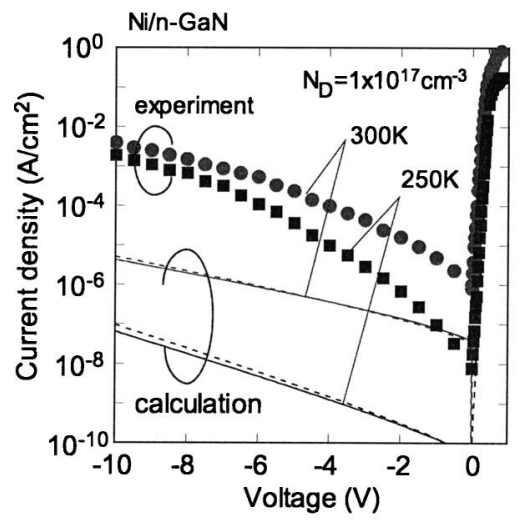

(b)

FIG. 2. (a) Theoretidal values of ideality factor, $\mathrm{n}_{\mathrm{F}}$, of the forward current of a Schottky diode with $\mathrm{SBH}=0.85 \mathrm{eV}$ as a function of temperature, calculated using Eq. (5) (solid lines), and calculated by the present computer program (symbols). (b) Theoretical $I-V$ curves calculated by approximate analytical formulas in Ref. 14 (solid curves), and by the present computer program (dashed curves) for a Schottky diode on uniformly doped $n$-GaN with $N_{D}=1 \times 10^{17} \mathrm{~cm}^{-3}$ and $\mathrm{SBH}=0.85 \mathrm{eV}$. For comparison, measured $I-V$ data are shown by closed circles and squares for a Ni/GaN Schottky diode with $N_{D}=1 \times 10^{17} \mathrm{~cm}^{-3}$.

transport is strongly by the TFE mechanism. As a result, the errors are enhanced in between two bias ranges, and an artificial connection of two analytical formulas produce artificial kinks in the theoretical curves, as discussed in Ref. 7.

In spite of the above unfavorable features, $I-V$ characteristics of highly doped $\mathrm{Si}$ and GaAs were reported to be well explained by the analytical formulas of Padovani and Stratton. ${ }^{14}$ In order to see whether these formulas are also applicable to Schottky barriers on highly doped GaN, reverse leakage currents data measured in our laboratory on a $\mathrm{Ni} / n-\mathrm{GaN}$ Schottky diode with a doping density of $N_{D}=1$ $\times 10^{17} \mathrm{~cm}^{-3}$ is also shown in Fig. 2(b) by closed circles and squares. It is seen in Fig. 2(b) that measured leakage currents are many orders of magnitude larger than the theoretical values calculated on the computer and by analytical formulas, and that the observed temperature dependence of current is much smaller than the theoretical prediction. Thus, a simple TFE/FE transport model cannot explain large reverse leakage currents in GaN Schottky diodes at all. 


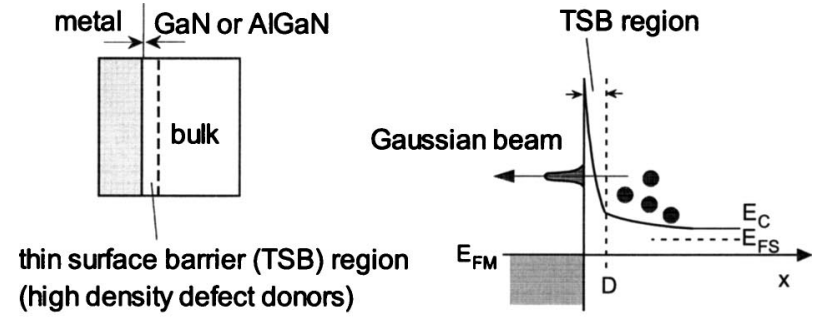

(a)
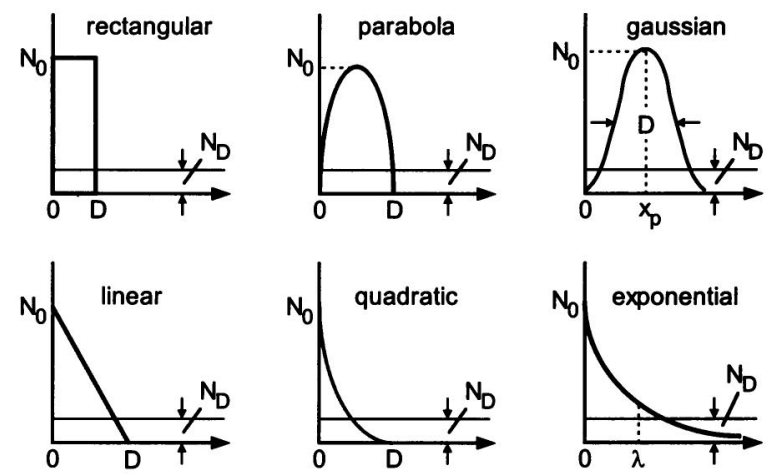

(b)

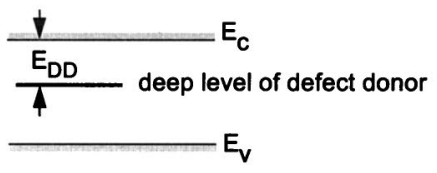

(c)

FIG. 3. (a) Schematic illustration of the TSB model. (b) Spatial density distributions of defect donors used for fitting. Density shapes as well as values of their characteristic parameters, $N_{0}, D$ and $\lambda$ were varied in the simulation. $N_{D}$ is the background uniform doping level of shallow donors. (c) Energy band diagram for the defect donor where the energy depth $E_{\mathrm{DD}}$ was varied in the simulation.

\section{TSB model}

In order to explain anomalously large leakage currents observed in GaN and AlGaN Schottky diodes, Hasegawa and Oyama ${ }^{7}$ have recently proposed a TSB model schematically shown in Fig. 3(a). The model assumes formation of high densities of unintentional defect donors near surface which reduces the width of the Schottky barrier, thereby enhancing the electron tunneling through the thin surface barrier in both forward and reverse directions in the form of a Gaussian beam. Either TFE or FE mechanism becomes dominant, depending on the temperature, bias direction, and bias magnitude. Assuming a rectangular distribution of defect donors on top of the uniform bulk doping, a set of analytical formulas were derived, and they explained the behavior of anomalously large leakage currents remarkably well. ${ }^{7}$ However, detailed quantitative agreements between theory and experiment were not entirely satisfactory due to the use of a simple rectangular distribution for which only an analytical treatment was possible.

As for the origin of defect donors, we proposed in our previous article ${ }^{7}$ that the defect donor is due to $\mathrm{N}$ vacancy or its related complex. Whether it is a shallow donor or deep donor could not be decided by the previous calculation, since only the case of the shallow donor could be treated analytically. From the viewpoint of theoretical defect physics, Neugebauer and Van de Walle, ${ }^{15}$ and Boguslawski et al. ${ }^{16}$ have calculated energy levels of native point defects in GaN. They concluded that a simple $\mathrm{N}$ vacancy defect $\left(\mathrm{V}_{\mathrm{N}}\right)$ acts as a shallow donor. On the other hand, recent results of calculation by Yamaguchi and Junnarkar ${ }^{17}$ predicted that the $\mathrm{V}_{\mathrm{N}}$ defect can form an s-like deep donor with $E_{\mathrm{DD}}$ of about 0.3 $\mathrm{eV}$. Experimentally, our recent transient analysis of drain currents in gateless HFETs with various surface treatments has detected presence of a $\mathrm{N}$ vacancy related deep donor level ${ }^{8}$ with the energy depth of $0.37 \mathrm{eV}$ on the $\mathrm{Al}_{0.25} \mathrm{Ga}_{0.75} \mathrm{~N}$ surface. Thus, if the calculation of $I-V$ curves can also be made for various values of the energy depth of the defect donor, it will be beneficial to resolve this issue.

Thus, theoretical $I-V$ curves were calculated in this article for various possible distributions of the defect donor as well as for various energy depths of the defect donor so as to reproduce the experimental $I-V$ curves over a wide temperature range.

\section{Parameters for numerical simulation of $I-V-T$ curves}

As possible spatial density distributions of the defect donor near surface, fittings were attempted for various peaked and decaying distributions shown in Fig. 3(b) with characteristic parameters. As peaked distributions, a rectangular distribution, a parabola-shaped distribution, and a Gaussian distribution were investigated, and as decaying distributions from the surface, a linearly decaying distribution, a quadratically decaying distribution, and an exponentially decaying distribution were tried. Roughly speaking, if defects are introduced at a certain point of time during crystal growth, a peaked distribution is likely to occur, and if defects are introduced from the surface during crystal growth or during processing after crystal growth, a decaying distribution from the surface is more likely. For the exponential distribution, the following expression was used:

$$
N_{\text {deep }}(x)=N_{0} \exp (-x / \lambda) \text {. }
$$

As another important parameter, the energy depth, $E_{\mathrm{DD}}$ of the defect donor shown in Fig. 3(c) was also varied as a fitting parameter.

\section{RESULT OF FITTING AND DISCUSSION}

\section{A. GaN and AIGaN Schottky diode samples}

As the experimental data for fitting, we used $I-V$ data measured on $\mathrm{Ni} / \mathrm{GaN}$ and $\mathrm{Ni} / \mathrm{Al}_{0.15} \mathrm{Ga}_{0.85} \mathrm{~N}$ diodes over a wide range of temperature $T$ in our laboratory as well as those reported by other workers. The structure of our sample is shown in Fig. 4(a). In these samples, $n$-GaN and $n$-AlGaN layers were grown by metalorganic vapor phase epitaxy. A typical value of electron mobility at room temperature was $500 \mathrm{~cm}^{2} / \mathrm{V} \mathrm{s}$ for $\mathrm{GaN}$ and $100 \mathrm{~cm}^{2} / \mathrm{V} \mathrm{s}$ for $\mathrm{AlGaN}$. A ring 


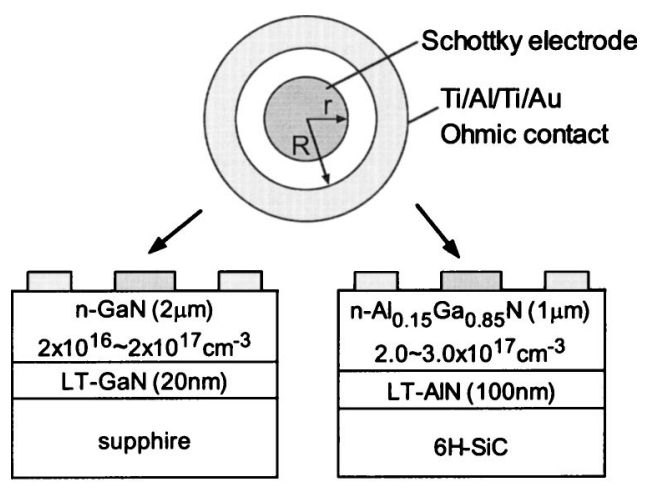

(a)

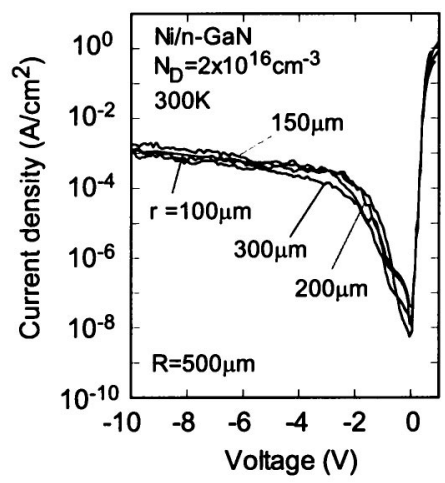

(b)

FIG. 4. (a) Structure of the sample used for measurements of $I-V$ curves. (b) Measured apparent current densities voltage for diodes having different radii of the Schottky electrode.

shaped ohmic contact was formed by standard photolithography on the $\mathrm{GaN}$ and $\mathrm{AlGaN}$ surface, using a $\mathrm{Ti} / \mathrm{Al} / \mathrm{Ti} / \mathrm{Au}$ $(20 / 80 / 20 / 50 \mathrm{~nm})$ alloy which was annealed at $600{ }^{\circ} \mathrm{C}$ for 2 min. Circular Schottky electrodes with a radius of $r$ $=100-300 \mu \mathrm{m}$ were formed at the center of the ohmic ring by a conventional electron-beam (EB) deposition process.

Since our samples had a lateral electrode configuration for the ohmic contact, there is a possibility that extra surface leakage currents flow in addition to bulk Schottky currents. To check the presence of such a possibility, $I-V$ characteristics were measured for samples having different areas. The result for $\mathrm{Ni} / n-\mathrm{GaN}$ Schottky diodes having different radii is shown in Fig. 4(b) in terms of the apparent current density defined as the measured current divided by the Schottky electrode area. The values of the apparent current density agree very well among samples with different radii, indicating that the measured apparent current densities can be regarded as the true current densities representing bulk Schottky barrier currents.

\section{B. Fitting for our GaN Schottky diodes}

First, we tried to reproduce the experimental data of our $\mathrm{Ni} / n-\mathrm{GaN}$ diode by calculating $I-V-T$ characteristics for various possible density distributions and various energy depths of the defect donor. It may look at first like various combinations of density functions, their parameters, and energy depths will reproduce the experimental data due to the

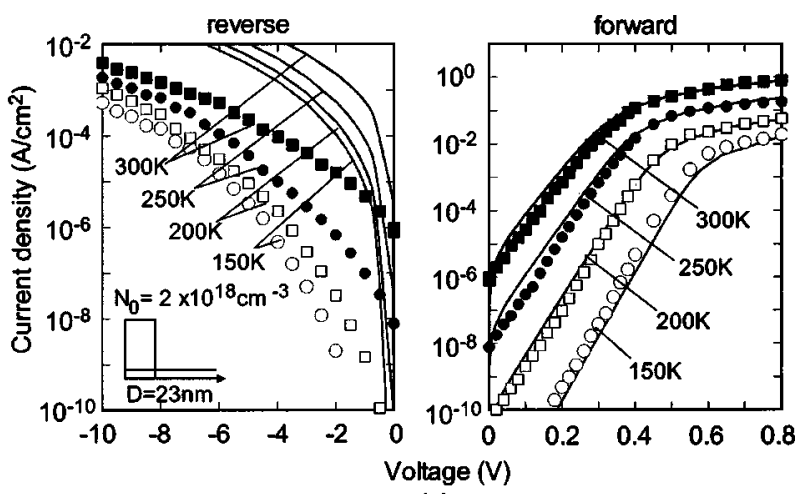

(a)

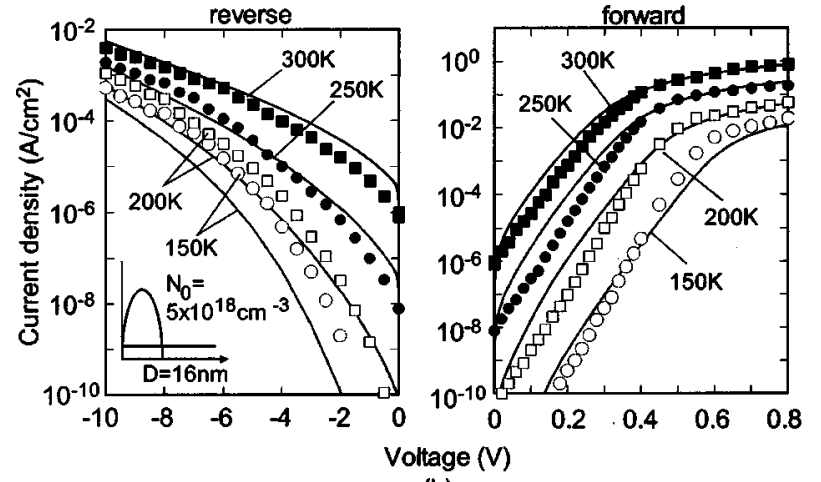

(b)

FIG. 5. (a) Calculated $I-V-T$ curves (solid curves) fitted to experimental data (symbols) of a $\mathrm{Ni} / n-\mathrm{GaN}$ diode, assuming a rectangular distribution with $N_{0}=2 \times 10^{18} \mathrm{~cm}^{-3}, D=23 \mathrm{~nm}$, and $E_{\mathrm{DD}}=0.25 \mathrm{eV}$. SBH=0.95 eV. (b) Calculated $I-V-T$ curves (solid curves) fitted to experimental data (symbols) of the same $\mathrm{Ni} / n-\mathrm{GaN}$ diode, assuming a parabola distribution with $N_{0}=5 \times 10^{18} \mathrm{~cm}^{-3}, D=16 \mathrm{~nm}$, and $E_{\mathrm{DD}}=0.25 \mathrm{eV} . \mathrm{SBH}=0.85 \mathrm{eV}$.

presence of many fitting parameters. However, it has turned out that it is not the case at all, and that successful fitting is possible only for one particular combination of distribution and energy depth, i.e., an exponentially decaying distribution and an energy depth of $E_{\mathrm{DD}}=0.25 \mathrm{eV}$, if one tries to reproduce the experimental data in both forward and reverse directions over a wide temperature range at the same time. This is probably because of the extreme sensitive nature of the tunneling process against the change of distribution shapes and slight variation of parameter values.

To show this, some fitting results are shown in the following. In all the fittings, the bulk shallow donor density $N_{D}$ was set to be that of the experimental sample of $N_{D}=1$ $\times 10^{17} \mathrm{~cm}^{-3}$. Figure 5(a) shows one of examples of the fitting into a rectangular distribution. The energy depth of $E_{\mathrm{DD}}=0.25 \mathrm{eV}$ was assumed. Here, series resistances of $0.5-13 \Omega \mathrm{cm}^{2}$ were introduced to fit the $I-V$ data in the high forward bias region. We used a rectangular distribution with $N_{0}=2 \times 10^{18} \mathrm{~cm}^{-3}$ and $D=23 \mathrm{~nm}$ so as to obtain a good fitting in the forward bias region. However, the results of calculation were then far away from the experiment in the reverse bias region, as seen in Fig. 5(a). A fitting example for a parabola distribution is shown in Fig. 5(b), assuming again $E_{\mathrm{DD}}=0.25 \mathrm{eV}$. A reasonably good fitting was obtained for forward direction for a parabola with $N_{0}=5 \times 10^{18} \mathrm{~cm}^{-3}$ and 


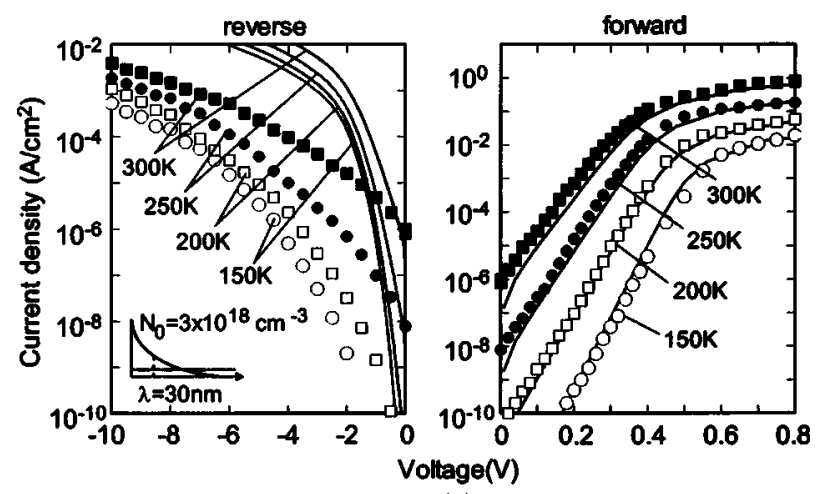

(a)

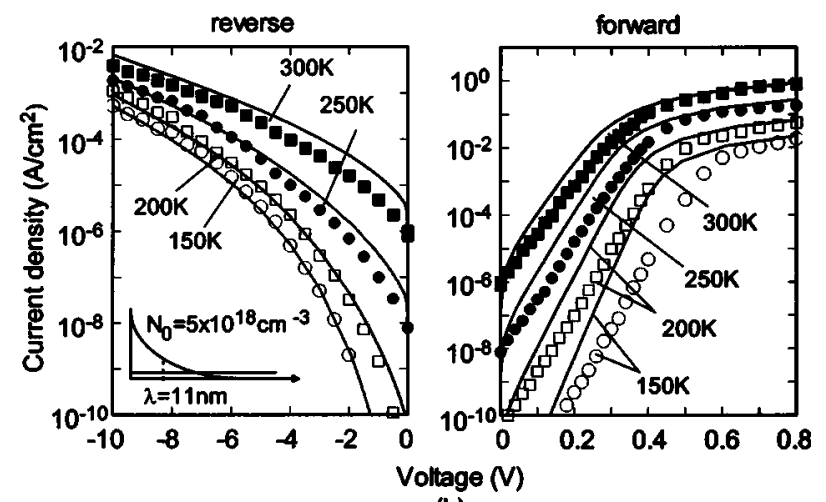

(b)

FIG. 6. (a) Calculated $I-V-T$ curves (solid curves) fitted to experimental data (symbols) of the $\mathrm{Ni} / n$-GaN diode, assuming an exponentially decaying distribution with $N_{0}=3 \times 10^{18} \mathrm{~cm}^{-3}, \lambda=30 \mathrm{~nm}$, and $E_{\mathrm{DD}}=0.01 \mathrm{eV}$. SBH $=0.85 \mathrm{eV}$. (b) Calculated $I-V-T$ curves (solid curves) fitted to experimental data (symbols) of the same $\mathrm{Ni} / n$-GaN diode, assuming an exponentially decaying distribution with $N_{0}=5 \times 10^{18} \mathrm{~cm}^{-3}, \lambda=11 \mathrm{~nm}$, and $E_{\mathrm{DD}}$ $=0.01 \mathrm{eV} . \mathrm{SBH}=0.85 \mathrm{eV}$.

$D=16 \mathrm{~nm}$. However, fitting for reverse $I-V-T$ characteristics was poor as shown in Fig. 5(b). Attempts using a Gaussian distribution gave similar poor results. Many trials for fitting indicated that none of the peaked distributions shown in Fig. 3(b) were suitable for reproducing the experimental $I-V-T$ curves, even if the distribution parameters and the energy depth of the defect level were changed over wide ranges.

As a representative of the decaying distribution, two fitting results for an exponentially decaying distribution with a shallow energy depth of $E_{\mathrm{DD}}=10 \mathrm{meV}$ are compared in Figs. 6(a) and 6(b). By assuming $N_{0}=3 \times 10^{18} \mathrm{~cm}^{-3}$ and $\lambda=30$ $\mathrm{nm}$, we could obtain a reasonably good agreement in the forward direction. For the reverse bias region, however, the calculation resulted in an extremely poor agreement, giving much larger magnitudes of currents with much weaker temperature dependence of currents than the experiment. Using a slightly different exponentially decaying distribution having $N_{0}=5 \times 10^{18} \mathrm{~cm}^{-3}$ and $\lambda=11 \mathrm{~nm}$ together with the same shallow energy depth of $E_{\mathrm{DD}}=10 \mathrm{meV}$, a reasonably good fitting was found to be possible in the reverse direction, as shown in Fig. 6(b). However, agreement in the forward direction became very poor.

After many trials, we obtained the best fitting result for
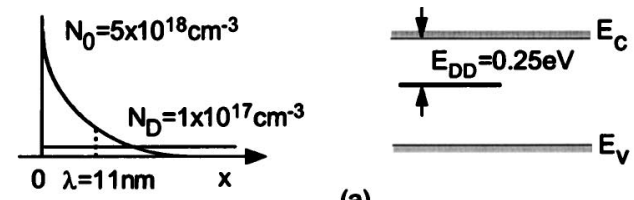

(a)
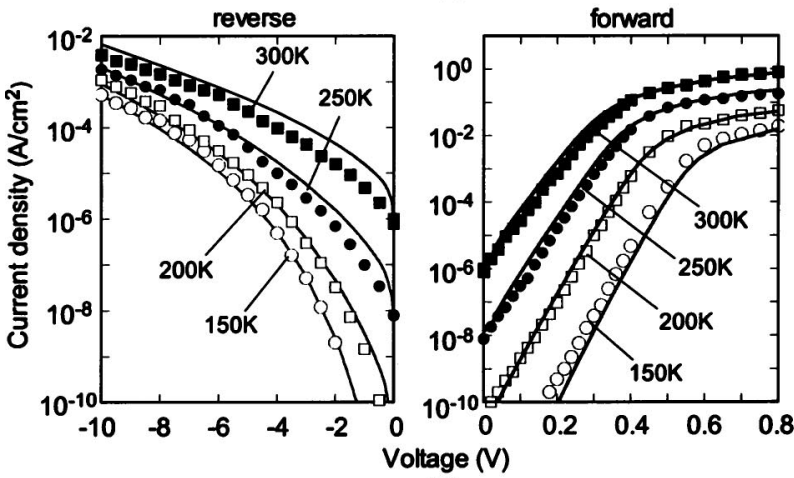

(b)
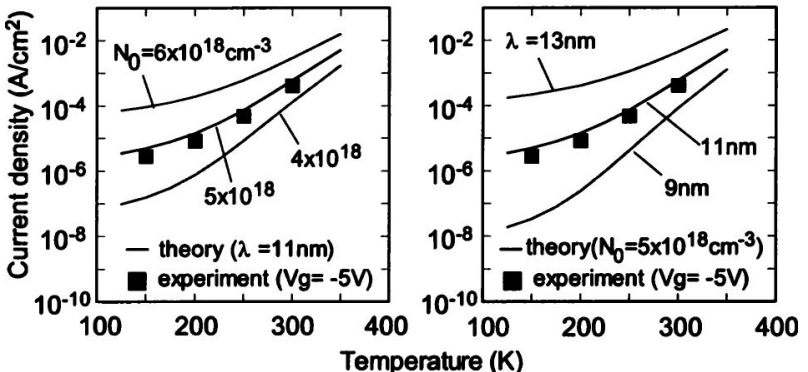

(c)

FIG. 7. (a) Best choice of distribution shape and parameter values used to reproduce the experimental data of our Ni/ $n-\mathrm{GaN}$ diode. $\mathrm{SBH}=0.85 \mathrm{eV}$. (b) The result of fitting for various temperatures. (c) Calculated parameter sensitivities of current density against $N_{0}$ and $\lambda$ of the exponential distribution.

the Ni/GaN Schottky diode by assuming an exponential decaying distribution shown in Fig. $7\left(\right.$ a) with $E_{\mathrm{DD}}=0.25 \mathrm{eV}$. The fitting result is shown Fig. 7(b). The simulation reproduced forward and reverse $I-V-T$ behavior almost completely. For the same set of experimental $I-V-T$ data used in this study, our previous analytical calculation ${ }^{7}$ for a rectangular distribution of surface defect donors superposed on bulk uniform doping, resulted in an equally good fitting with the experiment for the forward bias region. However, agreement was much poorer in the reverse direction. Furthermore, artificial connection of the approximate analytical formulas for FE and TFE transport produced artificial kinks in the theoretical curves for intermediate reverse biases ${ }^{7}$ similar to the case of uniform doping mentioned above. The present computer simulation solved all these problems.

It should be emphasized that, in order to achieve such good fitting for both forward and reverse directions simultaneously over a wide temperature range, defect donors should have a specific exponentially decaying distribution with a specific energy depth. For three types of decaying distributions of linearly decaying, quadratically decaying, and exponentially decaying distributions shown in Fig. 3(b), fitting improved with this order, and the best was obtained with the exponential distribution. Even for the exponential distribu- 

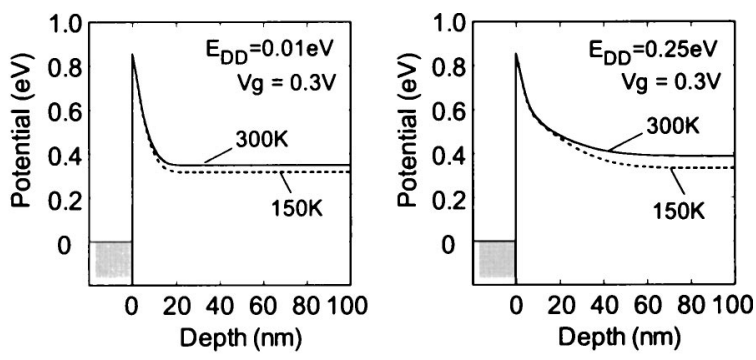

(a)

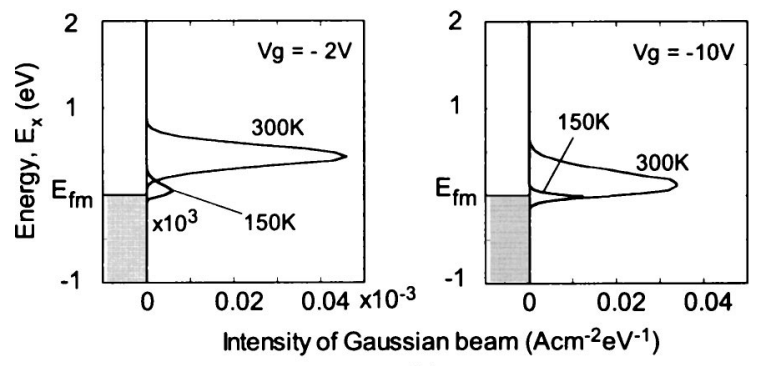

(b)

FIG. 8. (a) Calculated tunneling barrier profiles for the cases of $E_{\mathrm{DD}}$ $=0.01 \mathrm{eV}$ and $0.25 \mathrm{eV}$. (b) Calculated energy distributions of tunneling electrons for different biases and temperatures expressed in terms of the current density per unit energy.

tion, choice of the parameter values was very critical. To show this, the parameter sensitivities of reverse current density are plotted versus values of $N_{0}$ and $\lambda$ in Fig. 7(c) for various temperatures. The currents were very sensitive to choice of both $N_{0}$ and $\lambda$, especially in the low temperature region.

Similarly, it was found that the energy depth $E_{\mathrm{DD}}$ should be larger than $0.2 \mathrm{eV}$ and below $0.35 \mathrm{eV}$ to obtain acceptable fittings. The best value was $0.25 \mathrm{eV}$. Thus, the defect donor is a deep donor rather than a shallow donor. This value agrees with the theoretical value obtained by the s-like donor model for $\mathrm{N}$ vacancy. ${ }^{17}$ In order to understand how the energy depth of the defect donor can be determined by $I-V-T$ fitting, the shapes of the Schottky potential barriers were calculated for various values of energy depth. For example, calculated barrier shapes at the forward voltage of $0.3 \mathrm{~V}$ and at the temperatures of 150 and $300 \mathrm{~K}$ are compared in Fig. 8(a) for the cases of $E_{\mathrm{DD}}=0.01$ and $0.25 \mathrm{eV}$. The same exponentially decaying defect distribution shown in Fig. 7(a) with $N_{0}=5$ $\times 10^{18} \mathrm{~cm}^{-3}$ and $\lambda=11 \mathrm{~nm}$ was assumed. It should be noted that it is the same distribution used to obtain fitting shown in Fig. 6(b). In the case of $E_{\mathrm{DD}}=0.01 \mathrm{eV}$, almost the same thin barrier was formed at both temperatures due to the full ionization of shallow defect donor. This gave a larger magnitude and weaker temperature dependence of calculated forward currents than experimental ones, as seen in Fig. 6(b). On the other hand, a much larger change of barrier profile was seen in the case of $E_{\mathrm{DD}}=0.25 \mathrm{eV}$ due to reduction of the density of the ionized defect donors with decrease of temperature. This reduces tunneling current and increases its temperature dependence at low temperatures, leading to a good fitting of forward current shown in Fig. 7(b).
As for the reverse currents, the main feature of both of experimental and calculated currents shown in Fig. 7(b) is that they show a large temperature dependence at small biases, and temperature dependence is remarkably reduced as the reverse bias is increased. To understand this behavior, the energy distribution of electrons was calculated for different biases in terms of current density per unit energy as shown in Fig. 8(b). In all cases, distributions are nearly of Gaussian shape, as mentioned previously. However, its peak position is dependent on bias and temperature. When the reverse bias is small, the peak position is considerably above the metal Fermi level at $300 \mathrm{~K}$, showing TFE transport, and it approaches the metal Fermi level at $150 \mathrm{~K}$, leading to FE transport. On the other hand, the peak is always close to the metal Fermi level even at $300 \mathrm{~K}$ at large biases, and this gives a weak temperature dependence resulting from the FE transport. Thus, the anomalously large and weakly temperature dependent reverse leakage current in GaN Schottky barriers is mainly due to field emission from the metal Fermi level into the semiconductor at large reverse biases through a thin surface barrier which is further thinned down by a strong electric field.

\section{Fitting for GaN Schottky diodes reported in literature}

We also attempted to fit our calculation to experimental $I-V$ data of various GaN Schottky diodes reported by other groups.

Figure 9(a) shows fitting results to the experimental data on three different Pt/GaN diodes, $S_{1}, S_{2}$, and $S_{3} . S_{1}$ is after Guo et al., ${ }^{1} S_{2}$ is after Schmitz et al., ${ }^{2}$ and $S_{3}$ was prepared in our laboratory. Pt was deposited by EB deposition for three diodes. Excellent fitting was obtained for all three $I-V$ data by assuming the exponentially decaying distributions shown on the right of Fig. 9(a). The same energy depth of $E_{\mathrm{DD}}=0.25 \mathrm{eV}$ was used.

For comparison, the reverse current for the case of no defect donor $\left(N_{0}=0\right)$ is shown by a dashed curve for the case of $N_{D}=1 \times 10^{17} \mathrm{~cm}^{-3}$. The experimental currents are 7-8 orders of magnitude larger than the ideal case in all cases at high reverse biases. Detailed behavior of currents at low biases is, however, considerably different among three diodes. To understand this, the calculated energy distributions of tunneling electrons at a relatively small bias of -1.5 $\mathrm{V}$ are shown in Fig. 9(b). In the case of the $S_{1}$ diode, the GaN surface possesses the highest density of defect donors with the shallowest distribution. This resulted in the thinnest barrier width, leading to the highest Gaussian peak at a relatively high energy position, giving a large leakage current even at low biases. On the other hand, lower and wider distributions of defect donors in $S_{2}$, and $S_{3}$ diodes results in much smaller Gaussian peak heights, leading to smaller currents. It is seen that slight change in the defect donor distribution sensitively changes the height and position of Gaussian peaks, indicating that details of distribution of the surface defect donors play a dominant role in determining leakage behavior of GaN Schottky diodes. 

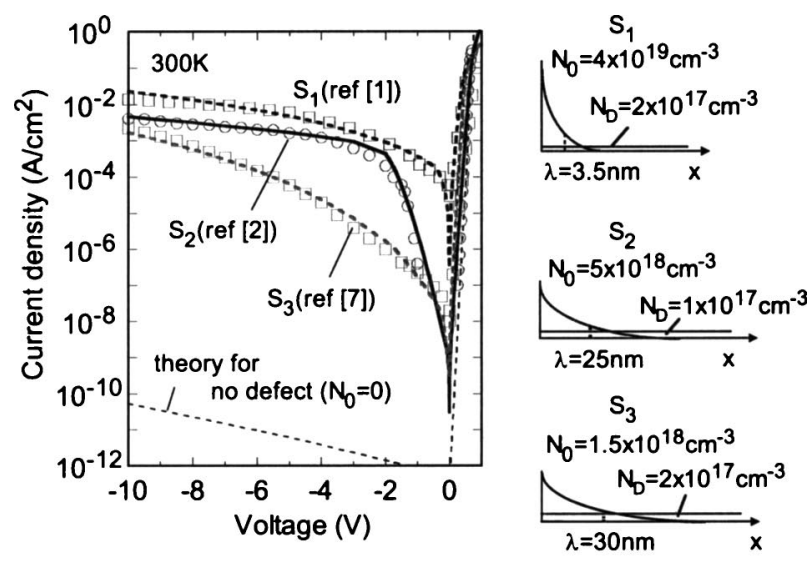

(a)

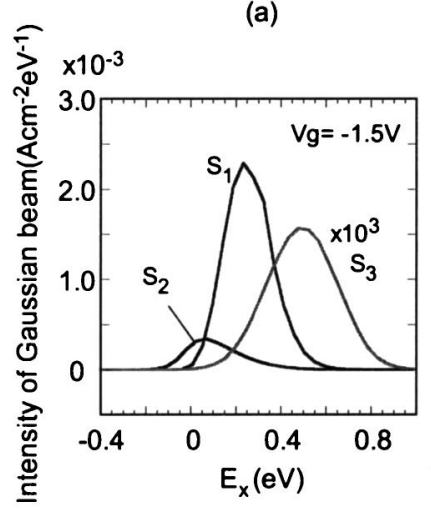

(b)

FIG. 9. (a) Calculated $I-V$ curves (solid curves) fitted to experimental $I-V$ data (symbols) of three different $\mathrm{Pt} / n-\mathrm{GaN}$ Schottky diodes produced in other laboratories $\left(S_{1}\right.$; Ref 1 and $S_{2}$; Ref 2$)$ as well as in ours $\left(S_{3}\right)$. Distributions of defect donors determined by best fitting are shown on the right. For comparison, calculated $I-V$ curve for the case of no defect $\left(N_{0}\right.$ $=0$ ) is also shown for $N_{D}=1 \times 10^{17} \mathrm{~cm}^{-3}$. (b) Calculated energy distributions of tunneling electrons at $V_{g}=-1.5 \mathrm{~V}$ for three diodes.

Figures 10(a) and 10(b) show another result of fitting of our theoretical curves to experimental forward $I-V-T$ curves of $\mathrm{Au} / n-\mathrm{GaN}$ and $\mathrm{Ag} / n-\mathrm{GaN}$ diodes reported by $\mathrm{Yu}$ et $a l .{ }^{10}$ and Sawada et al. ${ }^{18}$ respectively. For fitting, exponential distributions shown in the insets of Figs. 10(a) and (b) with the energy depth of $E_{\mathrm{DD}}=0.25 \mathrm{eV}$ were used. Again, our simulation completely reproduced these forward $I-V-T$ curves.

\section{Fitting for AIGaN Schottky diode}

The $I-V-T$ characteristics of $\mathrm{Al}_{0.15} \mathrm{Ga}_{0.85} \mathrm{~N}$ Schottky diodes were also investigated. As shown in Fig. 11(a), the fabricated $\mathrm{Ni} / \mathrm{AlGaN}$ diode exhibited large reverse leakage currents with strong bias dependence and very small temperature dependence. The result of fitting is also shown in Fig. 11(a). The spatial distribution and the energy depth of the surface defect donor, which gave the best fitting result, are shown in Fig. 11(b). Again, good fitting was possible only for an exponentially decaying distribution of deep donors. We used $E_{\mathrm{DD}}=0.37 \mathrm{eV}$ obtained by our transient measurements on gateless AlGaN/GaN HFETs ${ }^{8}$ which also turned out to be the best value for fitting.

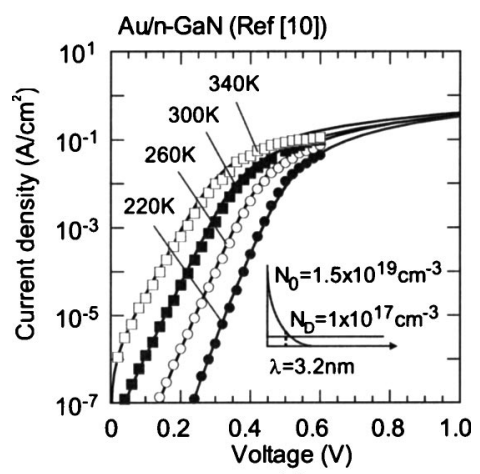

(a)

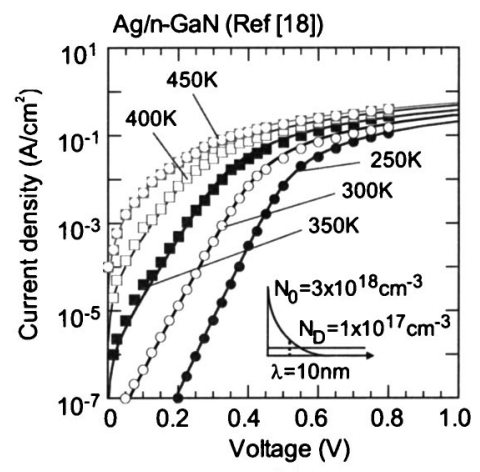

(b)

FIG. 10. Calculated forward $I-V$ curves (solid curves) fitted to experimental forward $I-V$ data (symbols) of $\mathrm{Au} / n-\mathrm{GaN}$ Schottky diodes reported by other workers (a) in Ref. 10 and (b) in Ref. 18. Distributions of defect determined by best fitting are shown in the insets.

An extremely high density of the defect donors seems to be present near the surface. This resulted in highly anomalous $I-V-T$ characteristics of the Schottky diode with excess leakage currents as observed as gate leakage in AlGaN/ GaN HFETs. ${ }^{9}$ The origin of the defect donor seems again to be $\mathrm{N}$ vacancy or its complex as discussed in Ref. 8 .

\section{E. Formation mechanism of defects and comparison with other models}

All the successful fitting obtained here had the following features: (1) The exponentially decaying distribution from surface always gave the best fitting. (2) The best energy depth was always the same, being $E_{\mathrm{DD}}=0.25 \mathrm{eV}$ for $\mathrm{GaN}$ and $0.37 \mathrm{eV}$ for $\mathrm{Al}_{0.15} \mathrm{Ga}_{0.85} \mathrm{~N}$. (3) The best values of $N_{0}$ and $\lambda$ of the exponential distribution were, on the other hand, very different from sample to sample, ranging from 1 $\times 10^{18}$ to $4 \times 10^{19} \mathrm{~cm}^{-3}$ for $N_{0}$ and $3-30 \mathrm{~nm}$ for $\lambda$ with a rough trend of large values of $N_{0}$ giving small values of $\lambda$. These features strongly indicate that the defect is the same for all the samples, being most likely $\mathrm{N}$ vacancy or its related complex, ${ }^{7,8,19}$ as mentioned already. These defects must be introduced from the surface either during crystal growth or during processing. In fact, we have previously detected the presence of a $\mathrm{N}$ vacancy related surface deep donors on the molecular beam epitaxy grown GaN surface by using a photoluminescence-based technique. ${ }^{20}$ But, the density was much smaller than the above numbers. The maximum pen- 


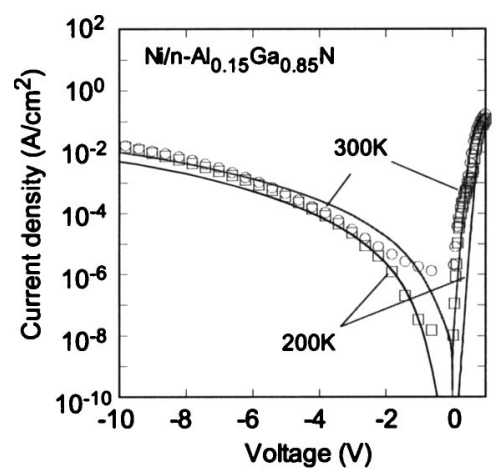

(a)

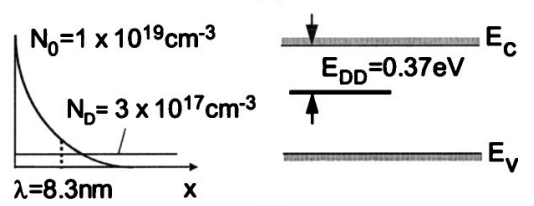

(b)

FIG. 11. (a) Calculated $I-V$ curves (solid curves) fitted to experimental $I-V$ data (symbols) of a $\mathrm{Ni} / n-\mathrm{Al}_{0.15} \mathrm{Ga}_{0.85} \mathrm{~N}$ Schottky diode. $\mathrm{SBH}=1.14 \mathrm{eV}$. (a) Best choice of distribution shape and parameter values used to obtain (a).

etration depth of $\lambda=30 \mathrm{~nm}$ also seems to be too large for the case of defect introduction during crystal growth.

As the formation mechanism of defect donors, we propose that the defect donors in the Schottky barriers are largely introduced during the process of deposition of metal by EB deposition, sputter deposition, or standard vacuum deposition where the surface is hit by high-energy metal atoms. In such cases, formation of process-induced deep levels depends on processing conditions, and can penetrate deep into the bulk. In fact, defect formation into depths deeper than $100 \mathrm{~nm}$ has been reported in the literature ${ }^{21-25}$ for EB deposition and sputter-deposition processes applied to GaAs and GaN. Thus, $\lambda=30 \mathrm{~nm}$ obtained by the present simulation is a very realistic value. The values of the integrated sheet density of defects given by $N_{0} \lambda$ were in the range of 4 $\times 10^{12}-1.4 \times 10^{13} \mathrm{~cm}^{-2}$ in our simulation, and these values again seem to be typical numbers for process-induced defects. The observed rough trend of large values of $N_{0}$ giving small values of $\lambda$ may be related to the thermal environment for processing such as substrate temperatures, annealing conditions, and cooling conditions. The exponentially decaying density distribution of defects, which was determined to be the most likely distribution by fitting, seems also to be consistent with the picture of processing induced defects. This is because, if the excess energy for defect formation carried by hot phonons, hot electrons, and/or energetic atoms, decays at a constant rate from surface into bulk, the distribution of density of defects which is proportional to energy loss, will also be an exponential one. Thus, the present simulation based on the TSB model together with metal-deposition induced formation of $\mathrm{N}$ vacancy or its complex seems to be capable of explaining all the aspects of experimental observation.

Finally, let us comment briefly on other models on reverse leakage currents in nitride based Schottky diodes. Several groups qualitatively suggested a trap-assisted tunneling mechanism for excess leakage currents in the reverse bias region. ${ }^{3,10,11}$ Here, the presence of discrete deep-level states or a continuum of trap states near the Schottky interface is assumed, and they are supposed to provide tunneling paths through the energy barrier after capture of electrons by thermal excitation. However, it is difficult to explain the observed large leakage currents by such a two-step or multistep process involving carrier capture by defects with small capture cross sections. Additionally, at low temperatures, the thermal excitation process of electrons from metal to higher trap levels would be suppressed and the current should be limited with the FE process. Experimentally, however, currents were far larger the prediction by the FE mechanism, as was shown in Fig. 2(b). Very recently, Karmalkar and co-workers ${ }^{12}$ tried to fit the gate leakage current of AlGaN/ GaN HFETs by the calculation based on the trap-assisted tunneling model under the unlikely assumption of a defect continuum with wide energy band widths. One has to assume a continuum with energy widths of $0.66-0.90 \mathrm{eV}$ extending throughout the depletion region of the semiconductor. This assumption is unacceptable because there has been no direct experimental evidence for the existence of such a peculiar defect continuum.

\section{SUPPRESSION OF REVERSE LEAKAGE CURRENTS}

According to the discussion in the previous section, highenergy metal atoms most likely cause the escape of $\mathrm{N}$ atoms from the surface during the metal deposition processes, and introduce N-vacancy-related deep donor levels near the Schottky interface. Based on this idea, we explored two possibilities of reducing leakage currents. One is the use of a low-energy electrochemical metal deposition process, and the other is to insert a high quality buffer layer at the Schottky interface to avoid direct bombardment by highenergy metal atoms.

Figure 12(a) shows the $I-V$ curves of $\mathrm{Pt} / n$-GaN Schottky diodes fabricated by a standard EB deposition process and by the electrochemical process whose details are given in Ref. 26. As mentioned in Sec. III B, the diode fabricated by the EB process exhibited large leakage currents at the reverse bias region due to the existence of high density of the surface defect donor. On the other hand, a remarkable suppression of leakage current was observed for the diode formed by the low-energy/low-temperature electrochemical process. The simulation of its $I-V$ curve showed that the electrochemical process can dramatically suppress the generation of the defect donors. Very recently, Miller and co-workers ${ }^{27}$ also reported about 3 orders reduction of reverse-bias leakage current in a $\mathrm{Ni} / n-\mathrm{GaN}$ diode by an electrochemical treatment in $\mathrm{NaOH}$ solution at $30^{\circ} \mathrm{C}$. However, they argued that formation of a thin oxide layer (Ga oxide) during the treatment was responsible for the reduction of leakage currents.

Suppression of leakage current was also attempted by insertion of an ultrathin $\mathrm{Al}_{2} \mathrm{O}_{3}$ passivation film which we have recently developed for $\mathrm{GaN}$ and $\mathrm{AlGaN}$ surfaces. ${ }^{28,29}$ The 


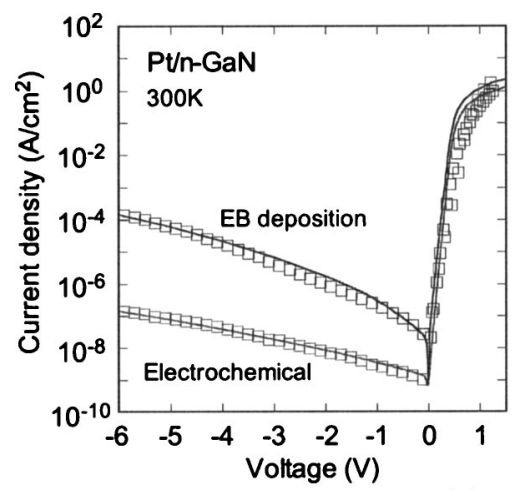

EB deposition

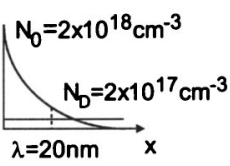

(a)

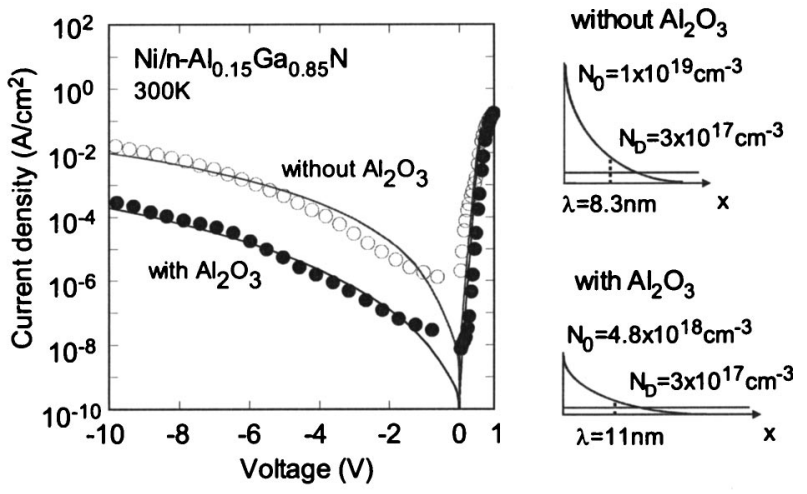

(b)

FIG. 12. (a) Experimental and calculated $I-V$ curves for Pt $/ n$-GaN Schottky diodes fabricated by a standard EB deposition process and by the electrochemical process. (b) Experimental and calculated $I-V$ curves of $\mathrm{Ni} / n-\mathrm{Al}_{0.15} \mathrm{Ga}_{0.85} \mathrm{~N}$ Schottky diodes with and without $1 \mathrm{~nm}$ thick $\mathrm{Al}_{2} \mathrm{O}_{3}$ passivation film. Distributions of defect donors determined by best fitting are shown on the right.

process sequence was as follows. First, the AlGaN surface was treated in the electron cyclotron resonance (ECR) $\mathrm{N}_{2}$ plasma at $280^{\circ} \mathrm{C}$ for $1 \mathrm{~min}$. This treatment partially recovers or terminates the $\mathrm{N}$ vacancy-related surface defects, leading to reduction of surface-defect traps and surface states on AlGaN. ${ }^{30}$ Then, an Al layer with a nominal thickness of $1 \mathrm{~nm}$ was deposited by molecular beam deposition at room temperature, followed by its oxidation using ECR-excited $\mathrm{O}_{2}$ plasma. Finally, the sample was annealed at $700{ }^{\circ} \mathrm{C}$ for 10 min under ultrahigh vacuum condition. Figure 12(b) compares the $I-V$ curves of $\mathrm{Ni} / n-\mathrm{Al}_{0.15} \mathrm{Ga}_{0.85} \mathrm{~N}$ Schottky diodes with and without $1 \mathrm{~nm}$ thick $\mathrm{Al}_{2} \mathrm{O}_{3}$ film. More than 2 orders of magnitude reduction of leakage current was observed for the diode with the $\mathrm{Al}_{2} \mathrm{O}_{3}$ passivation layer. The simulation result indicated that the $\mathrm{N}$ vacancy-related defect was greatly reduced by the insertion of a thin $\mathrm{Al}_{2} \mathrm{O}_{3}$ layer as shown on the right of Fig. 12(b).

\section{CONCLUSION}

Attempts were made to perform a rigorous computer simulation of the current transport in GaN and AlGaN Schottky diodes on the basis of the TSB model. First a simulation program for current transport of a Schottky barrier having an arbitrary potential profile was developed. Then, it was applied to calculation of $I-V$ curves for various spatial density distributions and various energy depths of defect donors on the basis of the TSB model. As a result, by assuming presence of $\mathrm{N}$ vacancy-related deep donors with an exponentially decaying density distribution from the surface, the simulation reproduced almost perfectly experimental $I-V-T$ curves of GaN and AlGaN Schottky diodes measured by our group as well as by other groups. This clearly proves the validity of the TSB model as the mechanism of anomalously large current leakage in nitride-based Schottky diodes. Attempts were also made to suppress surface defect donors at the GaN and AlGaN surfaces. A low-energy electrochemical metal deposition and a metal-insulator-semiconductor Schottky structure using an ultrathin $\mathrm{Al}_{2} \mathrm{O}_{3}$ film realized 2-3 orders of magnitude reduction of leakage currents, indicating very large reduction of deep defect donors.

\section{ACKNOWLEDGMENT}

This work is supported in part by the 21 Century COE program of "Meme-Media Technology Approach to the R\&D of Next-Generation ITs" from MEXT, Japan.

${ }^{1}$ J. D. Guo, M. S. Feng, R. J. Guo, F. M. Pan, and C. Y. Chang, Appl. Phys. Lett. 67, 2657 (1995)

${ }^{2}$ A. C. Schmitz, A. T. Ping, M. A. Kahn, Q. Chen, J. W. Yang, and I. Adesida, J. Electron. Mater. 27, 255 (1998).

${ }^{3}$ J. C. Carrano, T. Li, P. A. Grudowski, C. J. Eiting, R. D. Dupuis, and J. C. Cambell, Appl. Phys. Lett. 72, 542 (1998).

${ }^{4}$ X. A. Cao, S. J. Pearton, G. Dang, A. P. Zhang, F. Ren, and J. M. Van Hove, Appl. Phys. Lett. 75, 4130 (1999).

${ }^{5}$ E. Monroy et al., Phys. Status Solidi A 188, 367 (2001).

${ }^{6}$ S. Oyama, T. Hashizume, and H. Hasegawa, Appl. Surf. Sci. 190, 322 (2002).

${ }^{7}$ H. Hasegawa and S. Oyama, J. Vac. Sci. Technol. B 20, 1647 (2002).

${ }^{8}$ H. Hasegawa, T. Inagaki, S. Ootomo, and T. Hashizume, J. Vac. Sci. Technol. B 21, 1844 (2003).

${ }^{9}$ S. Mizuno, Y. Ohno, S. Kishimoto, K. Maezawa, and T. Mizutani, Jpn. J. Appl. Phys., Part 1 41, 5125 (2002).

${ }^{10}$ L. S. Yu, Q. Z. Liu, Q. J. Xing, D. J. Qiao, S. S. Lau, and J. Redwing, J. Appl. Phys. 84, 2099 (1998).

${ }^{11}$ E. J. Miller, X. Z. Dang, and E. T. Yu, J. Appl. Phys. 88, 5951 (2000).

${ }^{12}$ S. Karmalkar, D. M. Sathaiya, and M. S. Shur, Appl. Phys. Lett. 82, 3976 (2003).

${ }^{13}$ R. Stratton, J. Phys. Chem. Solids 23, 1177 (1962).

${ }^{14}$ F. A. Padovani and R. Stratton, Solid State Electron 9, 695 (1966).

${ }^{15}$ J. Neugebauer and C. G. Van de Walle, Phys. Rev. B 50, 8067 (1994).

${ }^{16}$ P. Boguslauski, E. L. Briggs, and J. Bernholc, Phys. Rev. B 51, 17255 (1995).

${ }^{17}$ E. Yamaguchi and M. R. Junnarkar, J. Cryst. Growth 189/190, 570 (1998).

${ }^{18}$ T. Sawada, Y. Ito, K. Imai, K. Suzuki, H. Tomozawa, and S. Sakai, Appl. Surf. Sci. 159-160, 449 (2000).

${ }^{19}$ H. Hasegawa and T. Hashizume, Mater. Res. Soc. Symp. Proc. 743, 45 (2003).

${ }^{20}$ M. Konishi, T. Hashizume, and H. Hasegawa, Inst. Phys. Conf. Ser. 170, 837 (2002)

${ }^{21}$ F. D. Auret, S. A. Goodman, G. Myberg, F. K. Koschnik, J. M. Spaeth, B. Beaumont, and P. Gibart, Physica B 273-274, 84 (1999).

${ }^{22}$ F. D. Auret, S. A. Goodman, F. K. Koschnik, J. M. Spaeth, B. Beaumont, and P. Gibart, Appl. Phys. Lett. 74, 2173 (1999).

${ }^{23}$ D. C. Look and Z. Q. Fang, Appl. Phys. Lett. 79, 84 (2001).

${ }^{24}$ R. Kleinhenz, P. M. Mooney, C. P. Schneider, and O. Paz, Proceedings of the 13th International Conference on Defects in Semiconductors, edited by L. C. Kimering and J. M. Parsey (Metallurgical Society of AIME, New York, 1985), p. 627.

${ }^{25}$ M. Nel and F. D. Auret, J. Appl. Phys. 64, 2422 (1988). 
${ }^{26}$ H. Hasegawa and T. Sato, J. Vac. Sci. Technol. B 15, 1227 (1997).

${ }^{27}$ E. J. Miller, D. M. Schaadt, E. T. Yu, P. Waltereit, C. Poblenz, and J. S. Speck, Appl. Phys. Lett. 82, 1293 (2003).

${ }^{28}$ T. Hashizume, S. Ootomo, T. Inagaki, and H. Hasegawa, J. Vac. Sci. Technol. B 21, 1828 (2003).
${ }^{29}$ T. Hashizume, S. Ootomo, and H. Hasegawa, Appl. Phys. Lett. 83, 2952 (2003).

${ }^{30}$ T. Hashizume, S. Ootomo, S. Oyama, M. Konishi, and H. Hasegawa, J. Vac. Sci. Technol. B 19, 1675 (2001). 